\title{
Lewis acid catalysis confined in zeolite cages as a strategy for sustainable heterogeneous hydration of epoxides
}

Weili Dai, ${ }^{1,2}$ Chuanming Wang, ${ }^{3}$ Bo Tang, ${ }^{1}$ Guangjun Wu, ${ }^{1,2}$ Naijia Guan, ${ }^{1,2}$ Zaiku Xie, ${ }^{3, \star}$ Michael Hunger, ${ }^{4}$ Landong $\mathrm{Li}^{1,2, *}$

${ }^{1}$ School of Materials Science and Engineering \& National Institute for Advanced Materials, Nankai University, Tianjin 300071, China.

${ }^{2}$ Key Laboratory of Advanced Energy Materials Chemistry of Ministry of Education, Collaborative Innovation Center of Chemical Science and Engineering, Nankai University, Tianjin 300071, China.

${ }^{3}$ SINOPEC Shanghai Research Institute of Petrochemical Technology, Shanghai 201208, China.

${ }^{4}$ Institute of Chemical Technology, University of Stuttgart, Stuttgart 70550, Germany.

* Corresponding author

Email:xzk@sinopec.com (Z.K.Xie) \&lild@nankai.edu.cn (L.D. Li) 


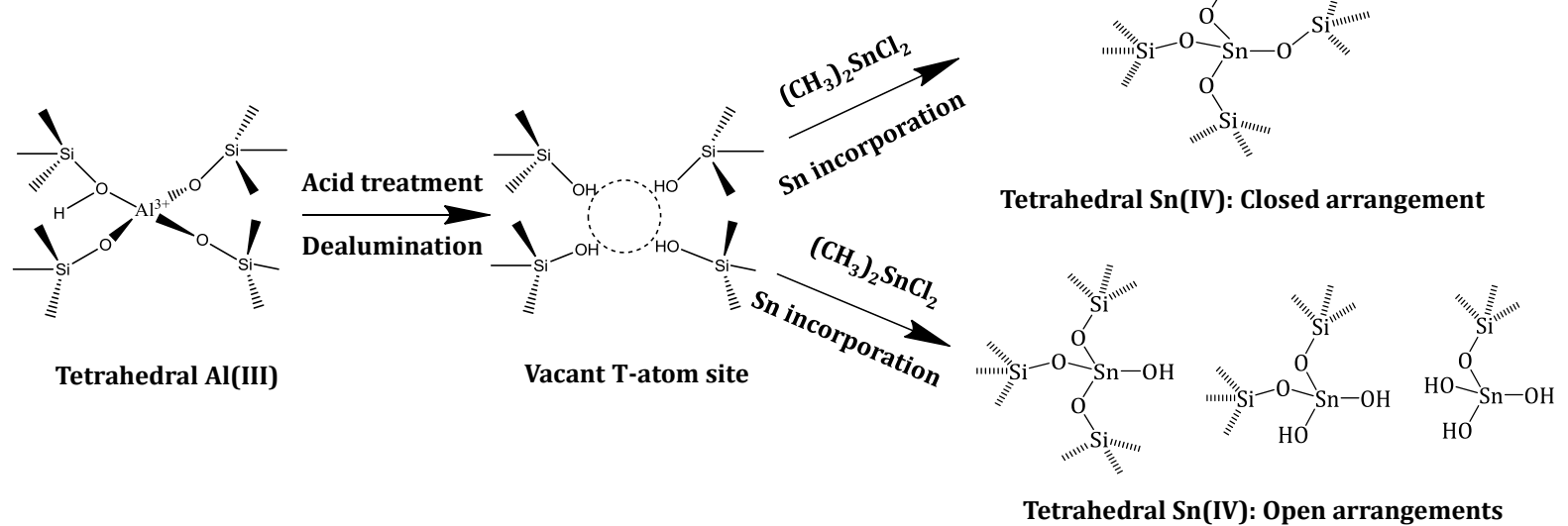

Scheme S1. Illustration of the incorporation of tetrahedrally coordinated Sn(IV) species into SSZ-13 zeolite with closed and open arrangements

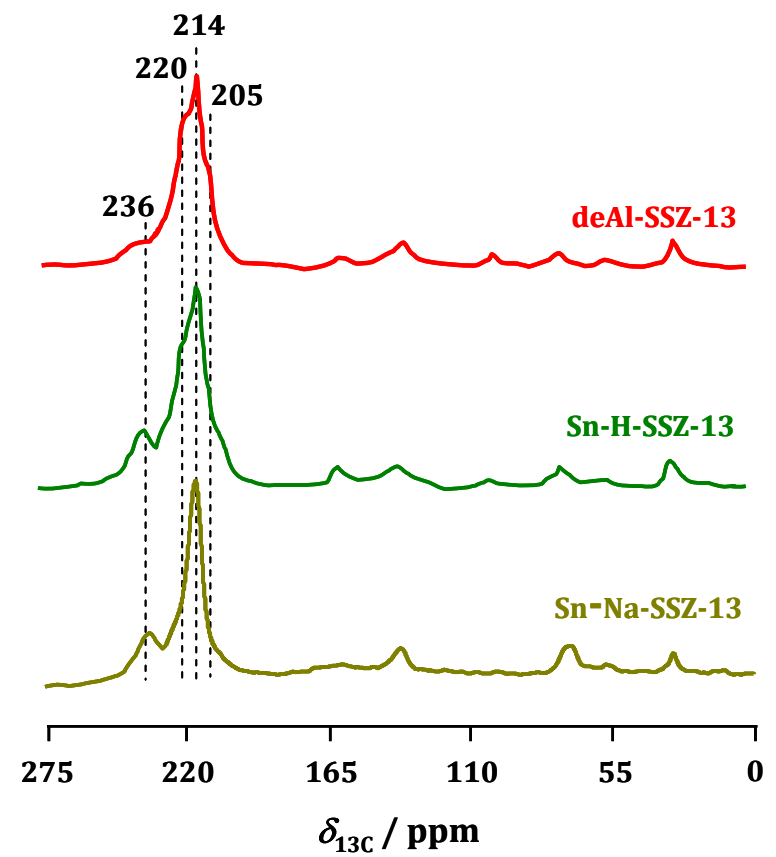

Figure S1. ${ }^{13} \mathrm{C}$ MAS NMR spectra of dehydrated samples recorded after loading of ${ }^{13} \mathrm{C}$-2-acetone on calcined samples. 

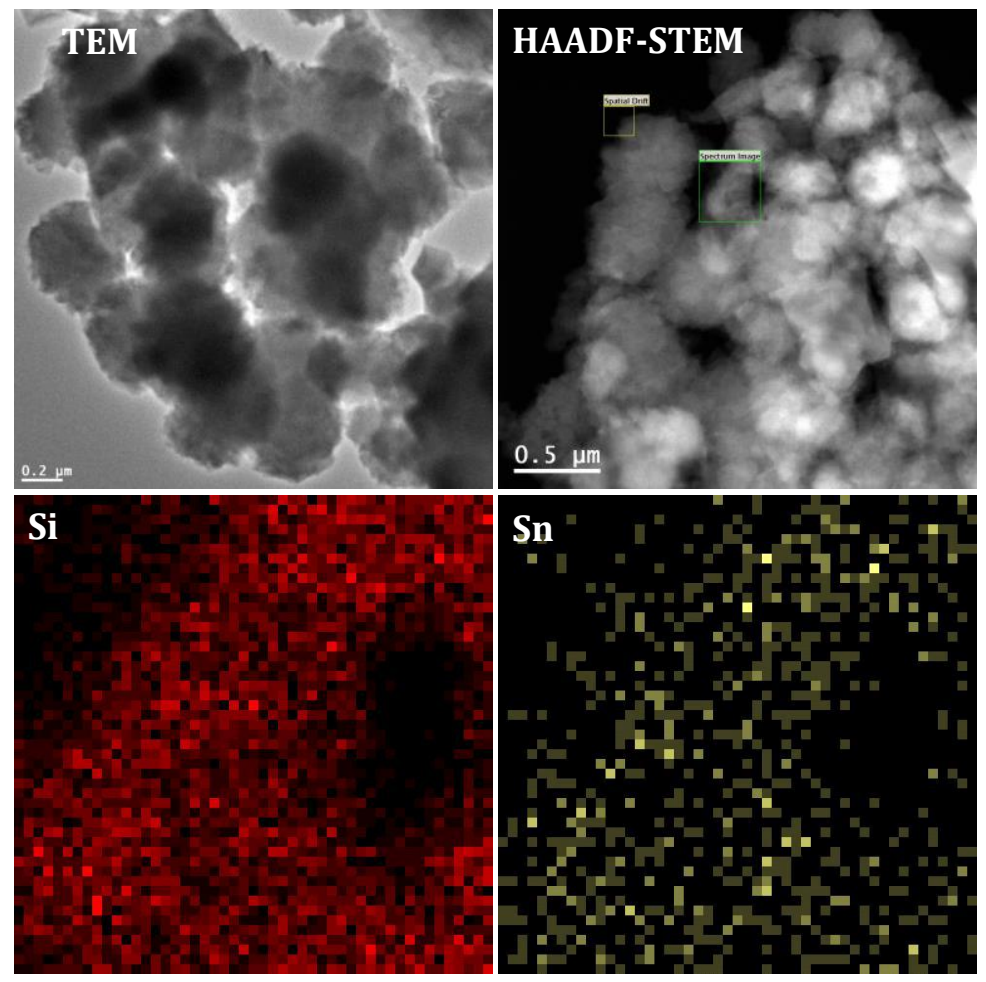

Figure S2. TEM and high angle annular dark field (HAADF) STEM images with corresponding elemental mapping
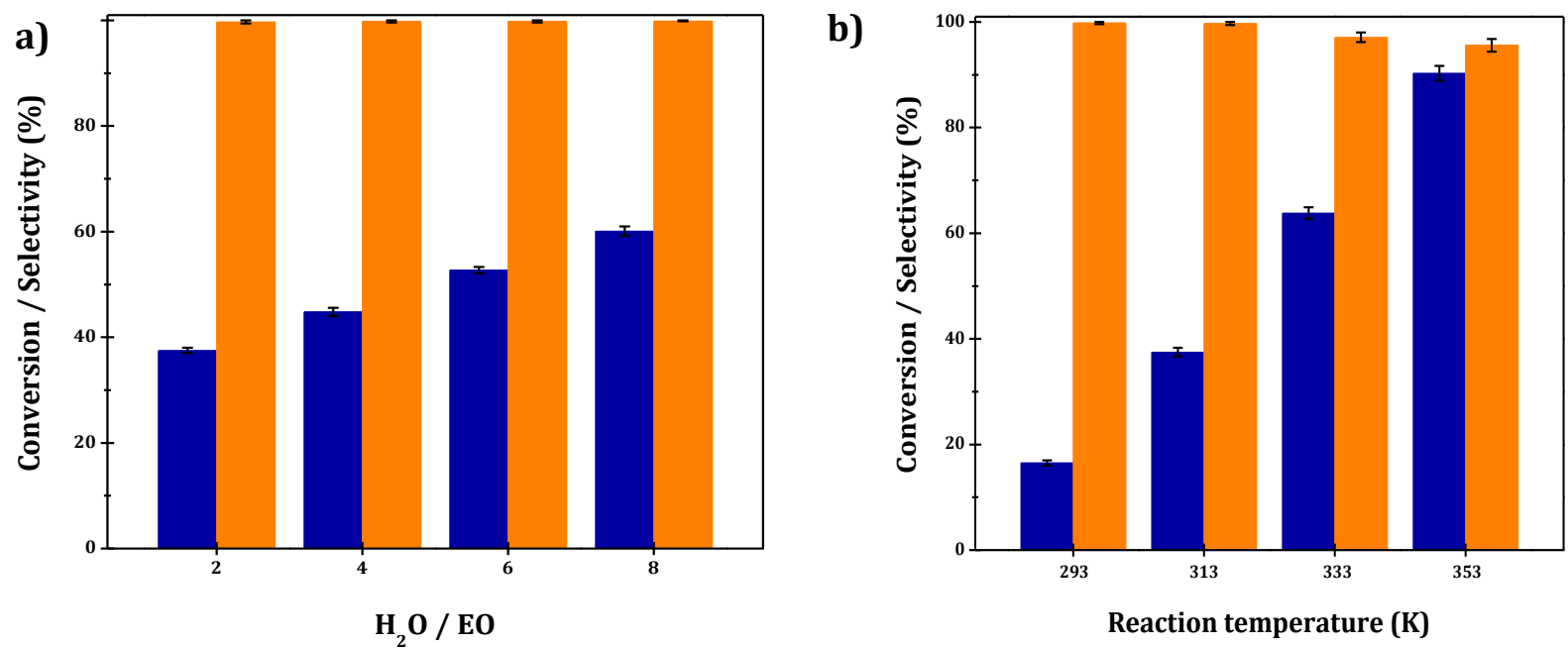

Figure S3. (a) Effect of $\mathrm{H}_{2} \mathrm{O} / \mathrm{EO}$ ratio on EO conversion and MEG selectivity catalyzed by Sn-H-SSZ-13. Reaction conditions: temperature $=313 \mathrm{~K}$; time $=4 \mathrm{~h}$. (b) Effect of reaction temperature on EO conversion and MEG selectivity catalyzed by Sn-H-SSZ-13. Reaction conditions: $\mathrm{H}_{2} \mathrm{O} / \mathrm{EO}=2$; time $=4 \mathrm{~h}$. 

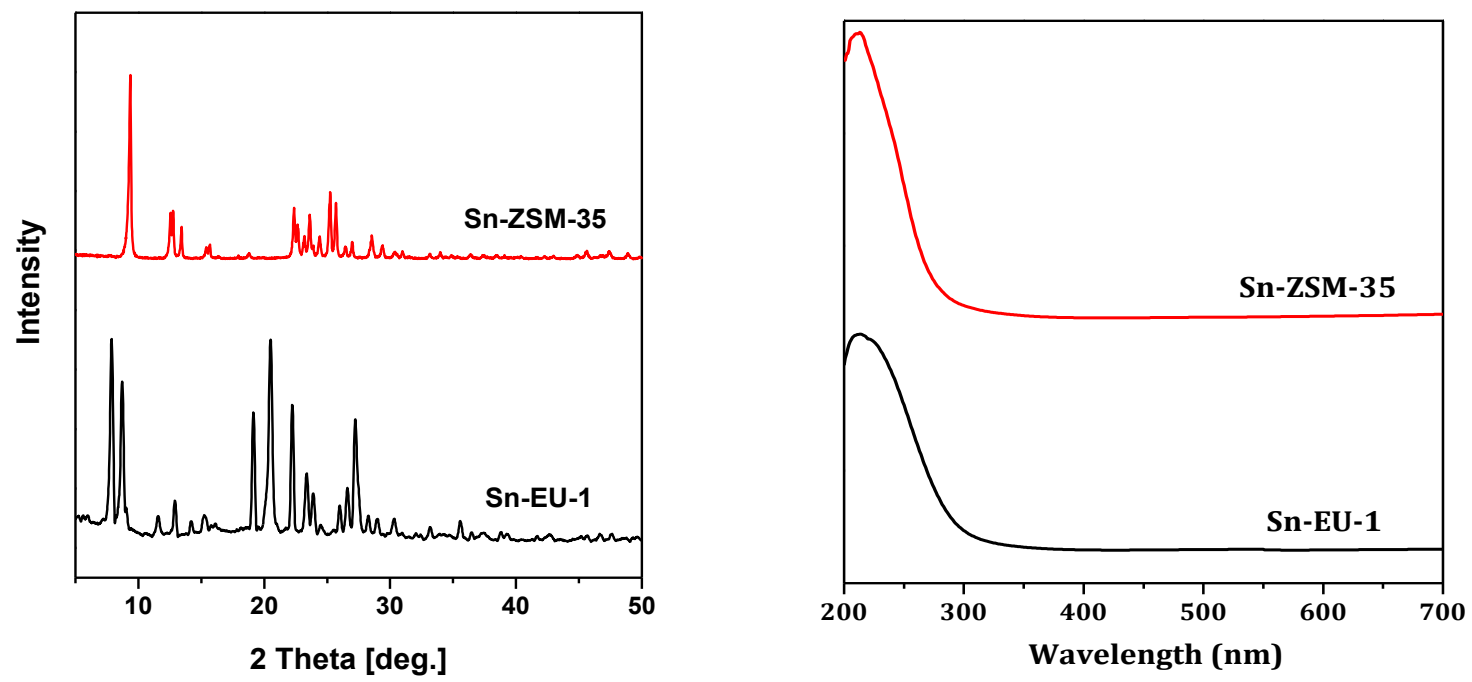

Figure S4. XRD patterns and UV-Vis spectra of Sn-H-ZSM-35 and Sn-H-EU-1

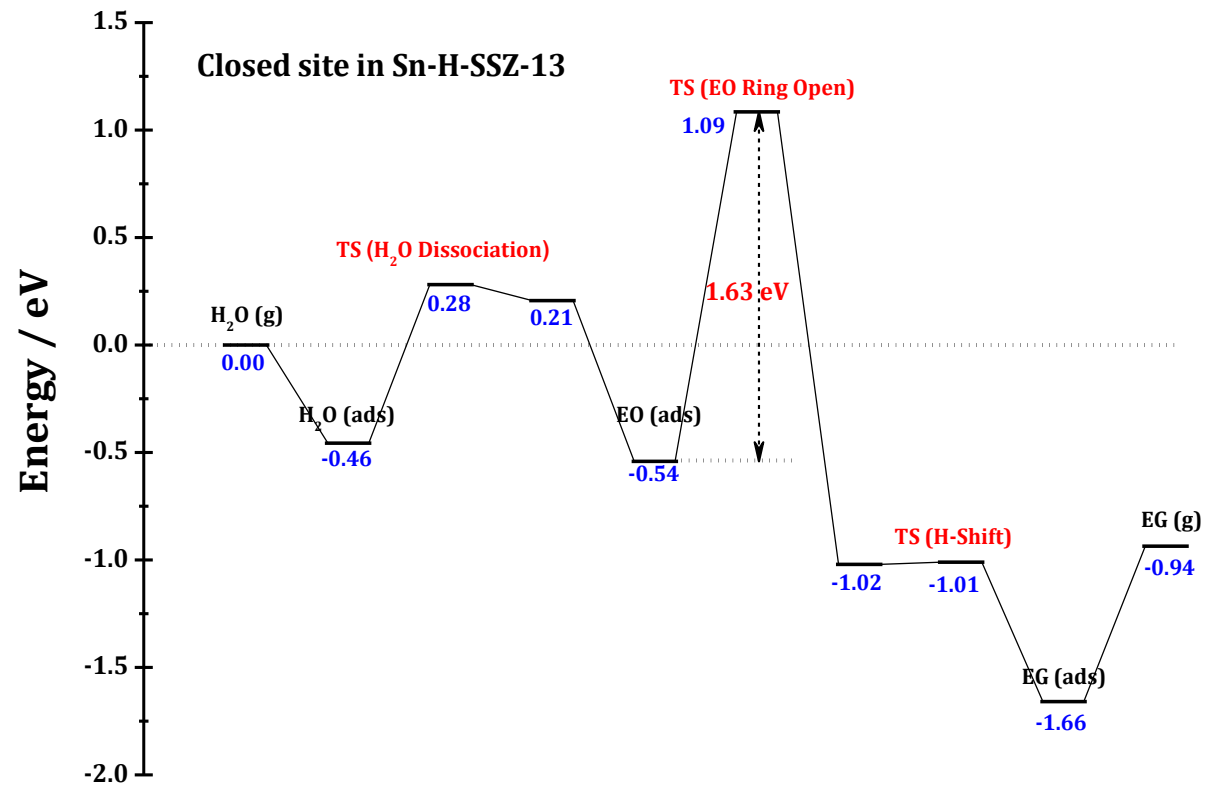

Figure S5. Energy profiles for EO hydration on closed site of Sn-H-SSZ-13.
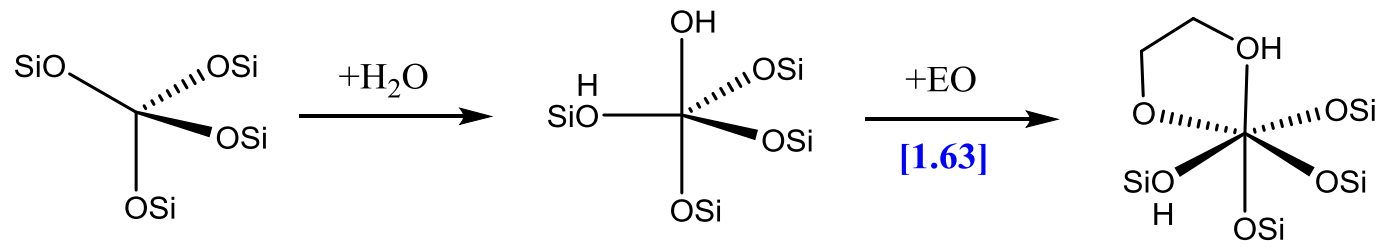

Figure S6. Schematic structures and energy barrier (eV) for the EO hydration in closed sites of Sn-H-SSZ-13 $\left[\mathrm{Sn}(\mathrm{OSi})_{4}\right]$. 

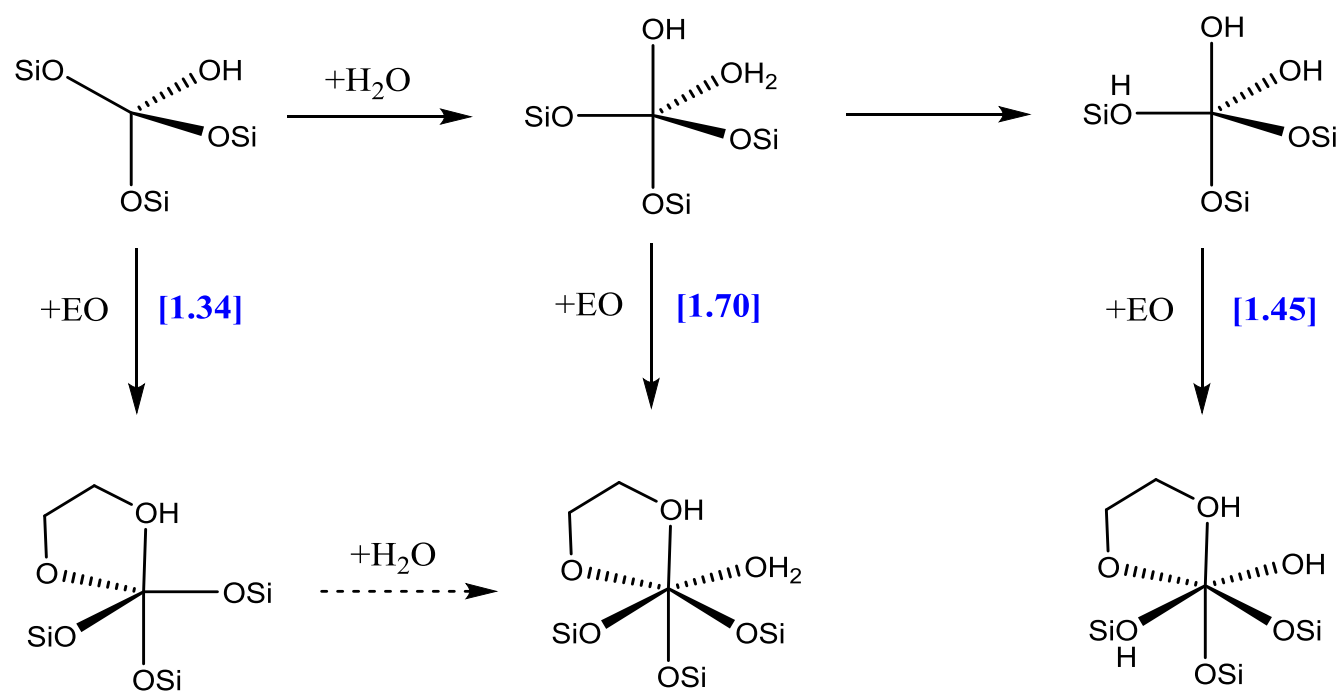

Figure S7. Schematic structures and energy barriers (eV) for the EO hydration in open sites of Sn-H-SSZ-13 $\left[\mathrm{Sn}(\mathrm{OSi})_{3}(\mathrm{OH})\right]$.
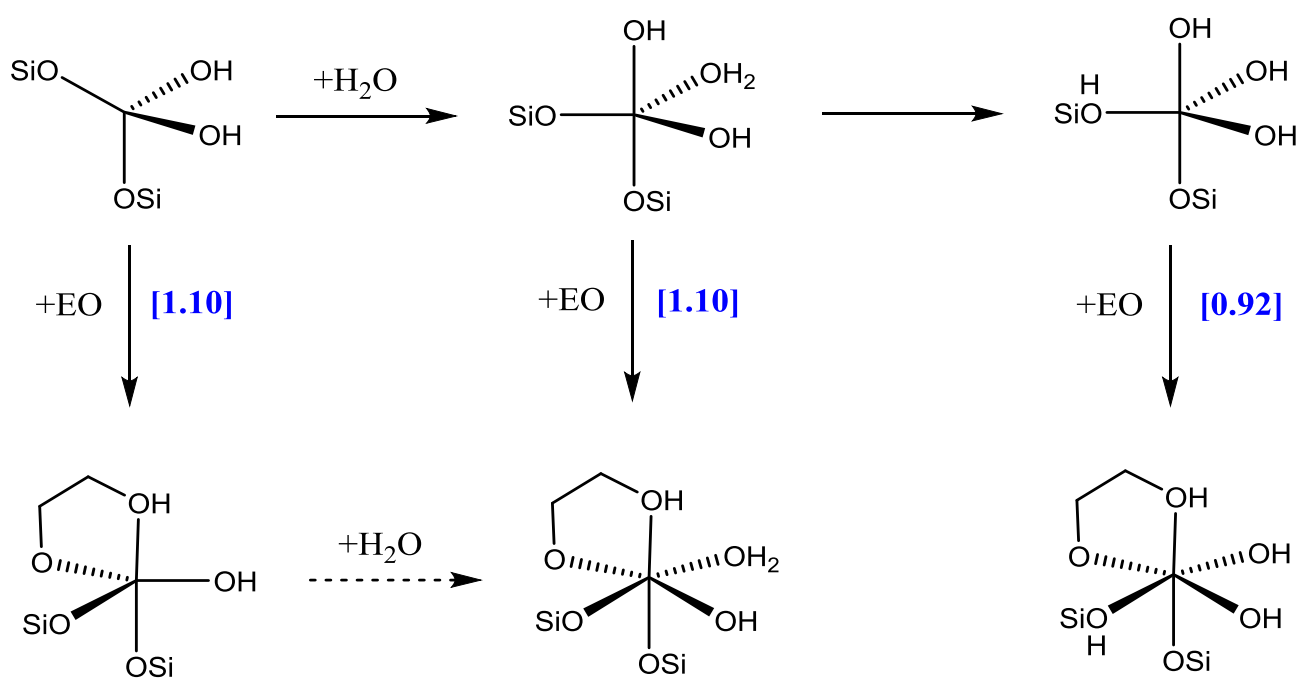

Figure S8. Schematic structures and energy barriers (eV) for the EO hydration in open sites of Sn-H-SSZ-13 $\left[\mathrm{Sn}(\mathrm{OSi})_{2}(\mathrm{OH})_{2}\right]$. 


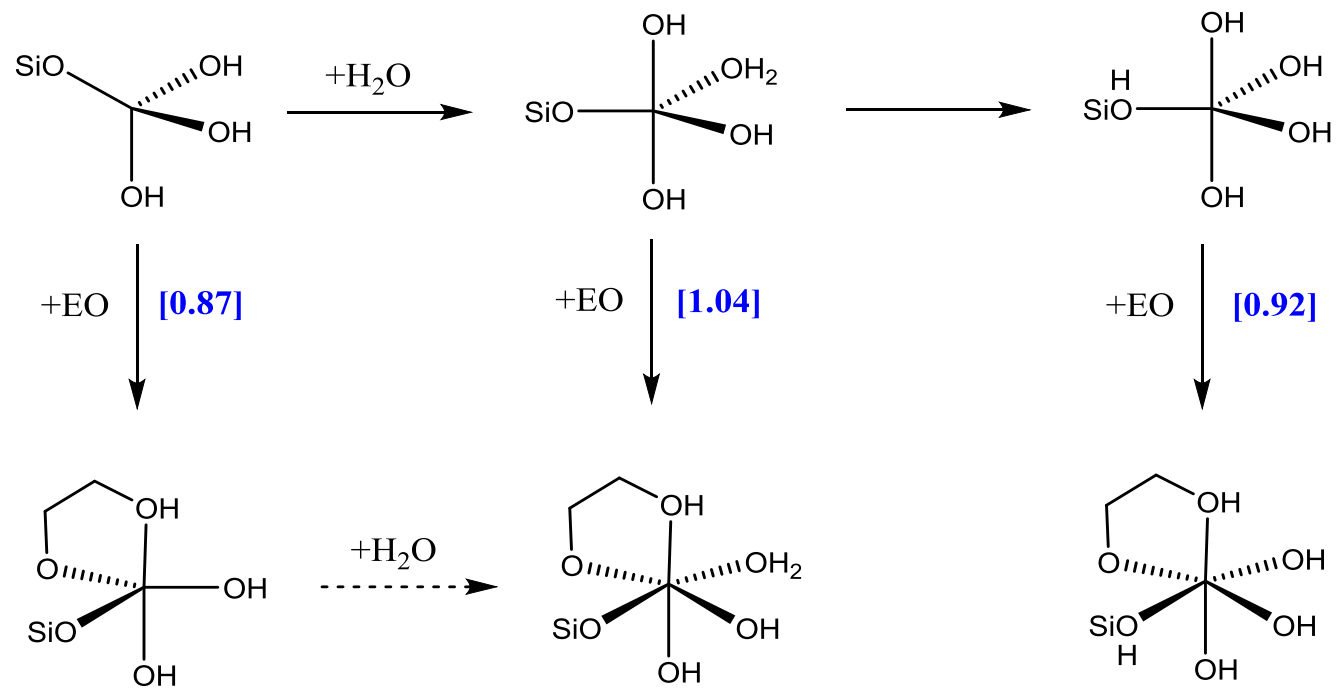

Figure S9. Schematic structures and energy barriers (eV) for the EO hydration in open sites of Sn-H-SSZ-13 $\left[\mathrm{Sn}(\mathrm{OSi})(\mathrm{OH})_{3}\right]$.
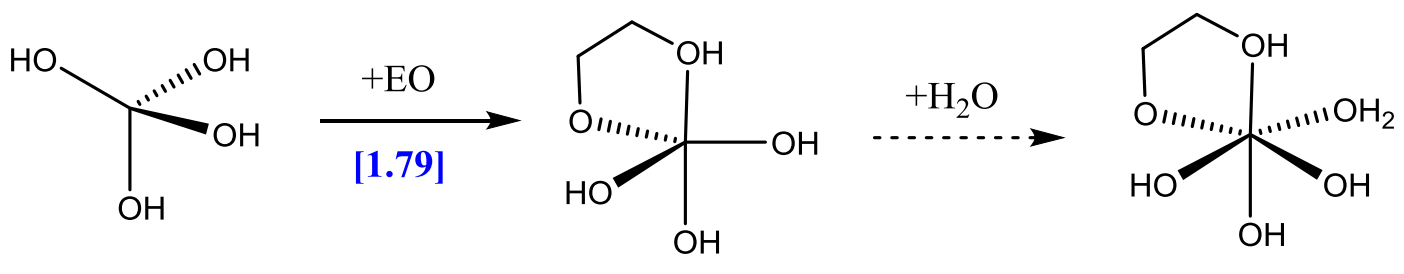

Figure S10. Schematic structures and energy barriers $(\mathrm{eV})$ for the EO hydration in open sites of Sn-H-SSZ-13 $\left[\mathrm{Sn}(\mathrm{OH})_{4}\right]$
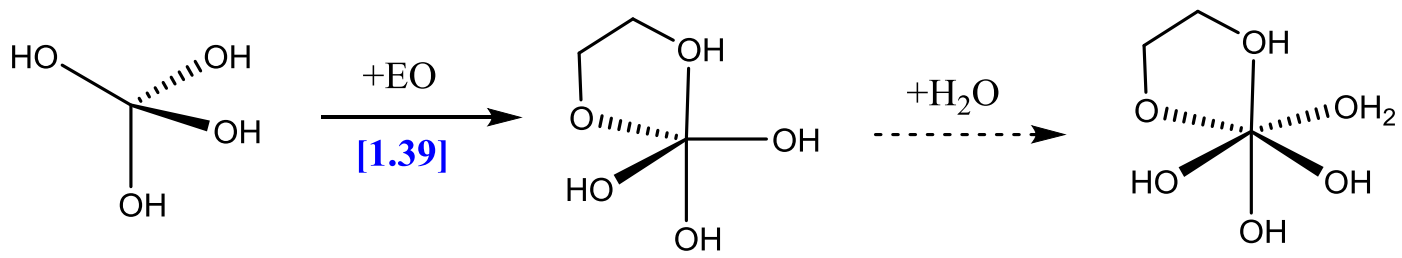

Figure S11. Schematic structures and energy barrier $(\mathrm{eV})$ for the EO hydration catalyzed by $\mathrm{Sn}(\mathrm{OH})_{4}$ in gas phase. 